\title{
Learning Task-Oriented Dexterous Grasping from Human Knowledge
}

\author{
Hui Li, Yinlong Zhang, Yanan Li, and Hongsheng $\mathrm{He}^{*}$
}

\begin{abstract}
Industrial automation requires robot dexterity to automate many processes such as product assembling, packaging, and material handling. The existing robotic systems lack the capability to determining proper grasp strategies in the context of object affordances and task designations. In this paper, a framework of task-oriented dexterous grasping is proposed to learn grasp knowledge from human experience and to deploy the grasp strategies while adapting to grasp context. Grasp topology is defined and grasp strategies are learned from an established dataset for task-oriented dexterous manipulation. To adapt to various grasp context, a reinforcement-learning based grasping policy was implement to deploy different task-oriented strategies. The performances of the system was evaluated in a simulated grasping environment by using an AR10 anthropomorphic hand installed in a Sawyer robotic arm. The proposed framework achieved a hit rate of $100 \%$ for grasp strategies and an overall top-3 match rate of $95.6 \%$. The success rate of grasping was $\mathbf{8 5 . 6 \%}$ during 2700 grasping experiments for manipulation tasks given in natural-language instructions.
\end{abstract}

Index Terms-Dexterous Grasping, Task-Oriented Grasping, Grasp Topology, Reinforcement Learning

\section{INTRODUCTION}

Robot dexterity plays an important role in accomplishing complex industrial tasks coping with a variety of objects in many applications, such as aerospace, automotive, manufacturing, warehousing and distribution [1]-[4]. Industrial robots are consistent and precise, but they are currently confined to structured production lines or simple and repetitive tasks. Robotic dexterity will enable complex tasks in unstructured environments.

Dexterous manipulation is one demanding function in many industrial applications. The existing robotic systems, however, lack the capability to determining proper grasp strategies in this context. Studies have shown that dexterous manipulation is related to object affordances and task designations [5]. Object affordance may include physical features of object such as dimensions, shape, and mass; task designations could include action, force, constrain, etc. Though there could be many possible ways to pick up an object, research has shown that human grasp topology trends to cluster over a large set of objects. Grasp taxonomy has been defined to simplify grasping choices. For example, a taxonomy of 16 grasping types was

Hui is with the Department of Electrical Engineering and Computer Science, The Wichita State University, USA

Yinlong is with Shenyang Institute of Automation, Chinese Academy of Sciences, China

Yanan is with the Department of Engineering and Design, University of Sussex, UK

*Correspondence should be addressed to Hongsheng $\mathrm{He}$, hongsheng.he@wichita.edu.

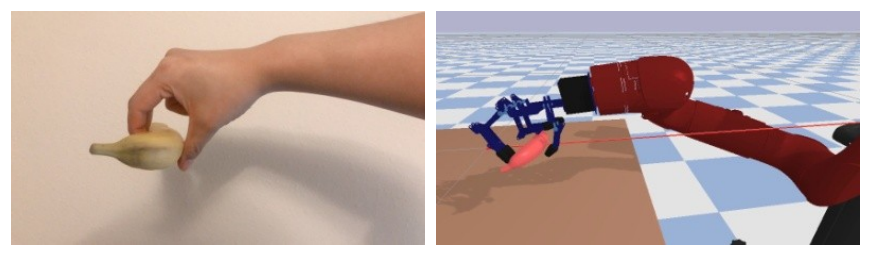

Figure 1. Robot accomplish task with grasp strategy learned from human knowledge.

created according to grasp strategies used by machinists in manufacturing environments [6]. Grasp taxonomy with 33 different grasp topology has also been proposed along the Yale human grasping dataset [7]. Except for grasping topology, little work has been done in achieving task-oriented grasping or leveraging human knowledge in grasping strategies.

In this paper, we proposed a task-oriented dexterous grasping approach, which can generate and deploy a proper grasping strategy based on task designation and object affordance. To represent the relation between object affordance, task designations, and human grasping strategy, a task-oriented object grasping dataset was developed, which enable learning grasping strategies of human experience. We designed a deep learning network to discover the human knowledge of grasping from the dataset. We also implemented a reinforcementlearning mechanism to deploy the selected grasping strategies. The learning-based grasping balances grasping flexibility and dexterity by adapting to various grasp context. The main contributions of the paper include

- We achieved the learning of human knowledge in grasping from the developed database; and

- We demonstrated the feasibility and effectiveness of dexterous grasping by using reinforcement learning while following grasping strategies.

\section{LEARNING GRASPING STRATEGIES}

Grasping strategies are related to object affordances and task designations [8]. The problem of grasp selection considering grasp context is a challenging problem, due to many influencing factors such as task representation, object property measurement, and the determination of a suitable grasp strategy. To solve this challenge, we implemented task-oriented dexterous grasping through learning human knowledge in grasping. We developed and trained a deep learning neutral network to predict proper grasp strategies. 


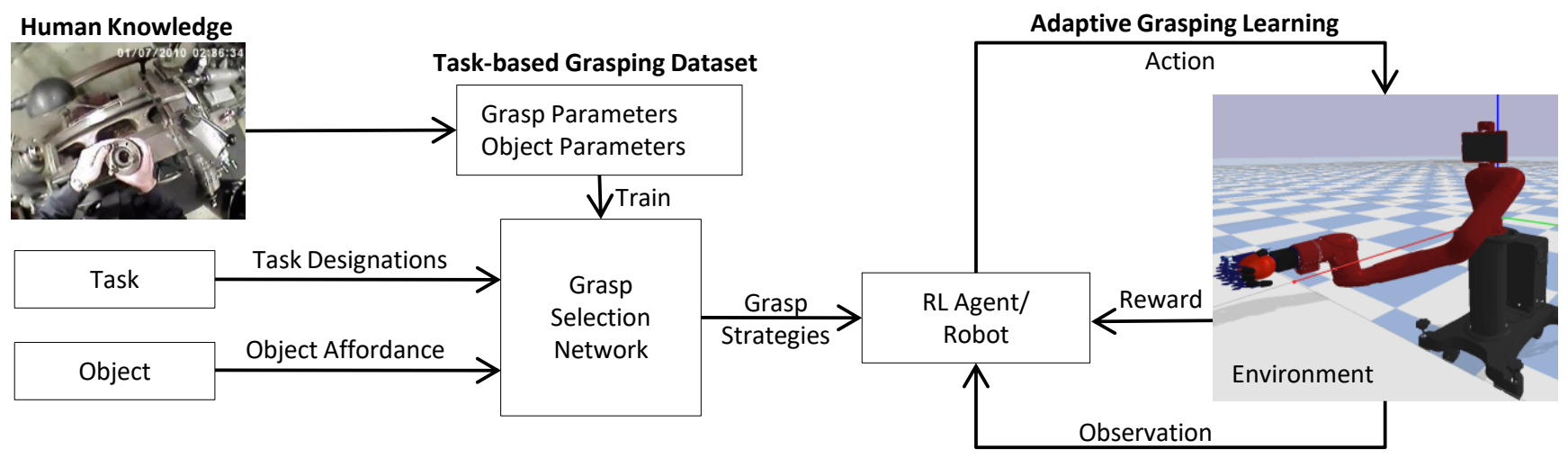

Figure 2. Work flow and task oriented grasp strategy generation system structure.

\section{A. Representing Human Grasping Knowledge}

We established a dataset for task-oriented object grasping based on the Yale human grasping dataset [7]. The Yale human grasping dataset was recorded by two housekeepers and two machinists about their regular work activities contains 27.7 hours of tagged video. A spreadsheet with 18210 entries were established based on the videos including related features of each task. The grasping topology was defined by grasp types, opposite types (pad, palm, side), and powerintermediate-precision (PIP). Tasks descriptions are given in natural-language descriptions containing action names, force (weight, interaction), constraints, and function class (hold, feel, use). Object affordances include physical properties of the object and grasped dimension along which an object is grasped.

The grasping tasks in the Yale dataset are redundant and ambiguous in task descriptions. We therefore refined the task list to 74 unique tasks by combining the tasks with similar actions. The dataset includes 33 unique types of grasp topology, and some are identical or similar. Thus, we reduced the types of grasping strategy to 15 as shown in Fig. 3 . The refined grasping dataset includes 15 grasp topology, 255 unique objects, 74 unique actions as shown in Fig. 4. The dataset is available online ${ }^{1}$. In addition, the Yale human grasping dataset does not provide $3 \mathrm{~d}$ models of objects. We collected similar objects and augmented the dataset with 157 3D object models.

\section{B. Learning Grasp Strategies}

We developed a parsing algorithm based on SpaCy to extract task designations from task descriptions. Task descriptions usually contain an action verb and a target object. Cosine similarity is computed between the extracted action and unique actions in the task-oriented object grasping dataset. The most similar actions are selected as the target et action, and the associated task parameters are also chosen. The object parameters were obtained based on our prior work [9], [10].

The task-oriented object grasping dataset is further processed to train the model. The categorical features in the dataset are converted to indicator values which expand the

${ }^{1}$ https://github.com/hhelium number of features in the dataset to 127 . The continuous features such as dimension and mass are normalized to improve robustness of the network. In real world scenario, more than one grasp topology could be suitable for a certain task. We therefore designed a multi-class multi-label grasp selection network to predict grasp topology. The network includes three subnetwork: the grasp topology selection network $g(f)$, the OppoType selection network $o(f)$ and the PIP selection network $p(f)$ where $f$ is the input features.

- The grasp type selection network $g(f)$ includes one input layer with 133 neurons, three hidden layers with $2^{11}, 2^{8}$ and $2^{6}$ neurons and a output layer contains 15 neurons. All units in the input and hidden layers are activated by ReLU while the output layer uses sigmoid activate function. The output of $g(f)$ is a set of probability distribution over 15 grasp types.

- The OppoType selection network $o(f)$ and the PIP selection network $p(f)$ have the same structure. They share the input layer and the first two layers of hidden layers with $g(f)$ and their output layer contains 3 neurons activated by softmax.

The object grasping dataset is split into two parts for training. The training and validation data contains $90 \%$ of grasping samples, and the test data includes the rest 10\% (650). The algorithm was trained with 4-fold cross-validation. The hyperparameters include a batch size 64, 5000 training epochs and a 0.001 learning rate for the Adam optimizer. We achieved training accuracy of $85 \%$ for $o(f)$ and $p(f)$, and a $100 \%$ hit rate for $g(f)$.

\section{AdAPtive GRASP DePloyment}

The predicted grasp strategies need to be deployed to grasp the object. Since the grasp size may vary depending on the shape, size or even rigidity of the object, we developed a deep reinforcement learning algorithm is trained to deploy adaptive grasps for different objects. Traditional grasping task requires complex calculation on path and grasp points, and it is not adaptive enough for Task-oriented grasping. In this paper, we design a reinforcement learning algorithm to learn adaptive grasp since reinforcement learning allows the robot to learn to grasp by itself and able to respond to unforeseen environments 


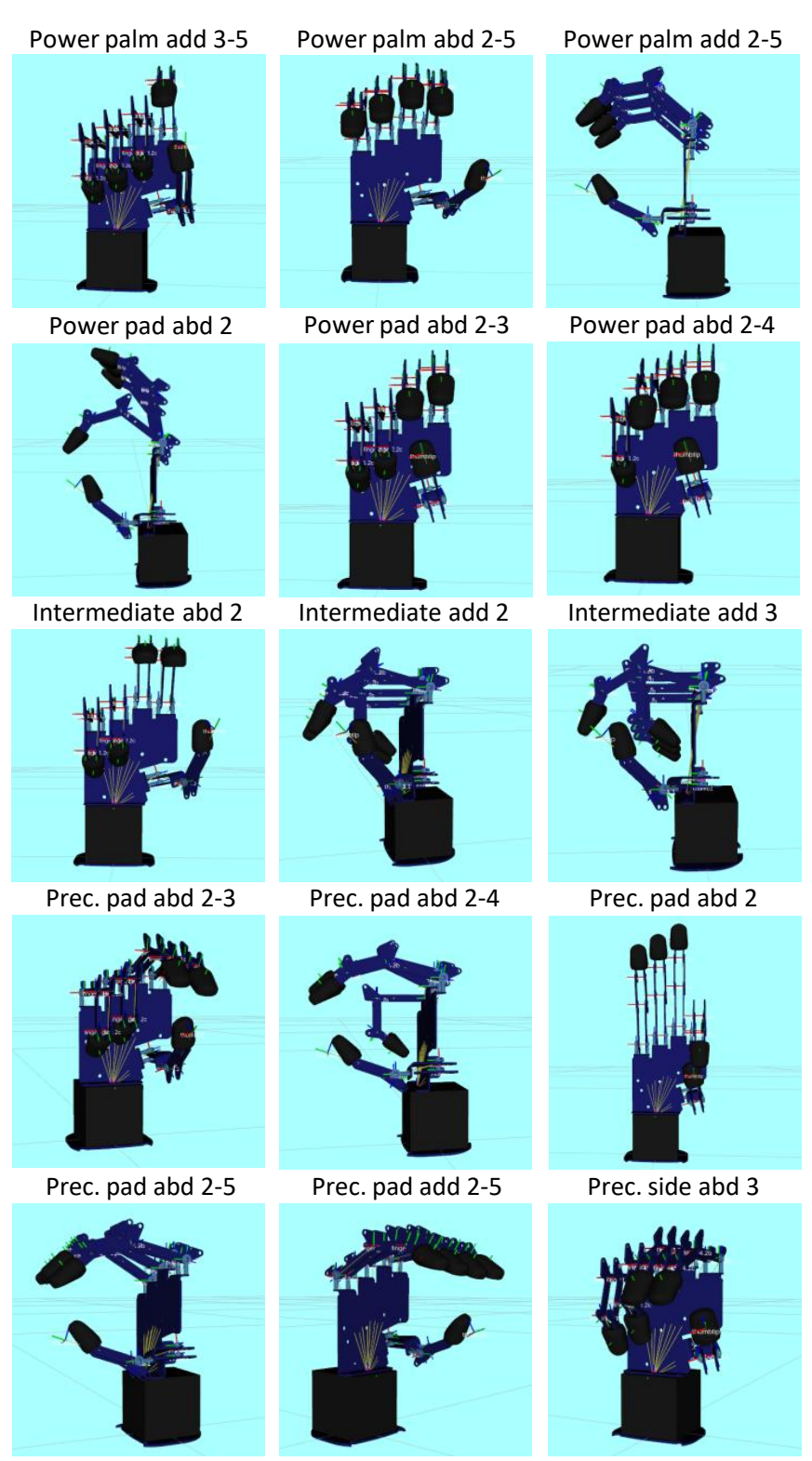

Figure 3. Grasp Types developed in simulation environment.

A Proximal Policy Optimization (PPO) reinforcement learning algorithm [11] is implemented using actor-critic policy [12] to teach the robot to control a robot to grasp and move a target object adaptively. PPO updates it's policy using previous experience

$\theta_{\text {new }}=\operatorname{argmax} E\left[\min \left(r_{t}(\theta) A_{t}, \operatorname{clip}\left(r_{t}(\theta), 1-\varepsilon, 1+\varepsilon\right) A_{t}\right)\right]$

where $\theta$ is policy parameter, $\theta_{\text {new }}$ is updated policy parameter, $E$ indicates empirical expectation over time steps, $r_{t}$ is the ratio between new policy and old policy. $A_{t}$ is advantage at time $t, \varepsilon$ is a small number usually 0.1 or 0.2 and clip will clip $A_{t}$ while $r_{t}$ is not between $1-\varepsilon$ and $1+\varepsilon$.

Since we focus on adaptive grasp deployment, fixed grasp path and object location are used in this work. Each joint in the hand can be controlled by a scaler $\alpha_{i} \in[0,1]$. The joint will fully extent when $\alpha_{i}=0$ and completely close while $\alpha_{i}=1$. The AR10 hand has 10 joints, the action function is $a=$ $\left[\alpha_{0}, \alpha_{1}, \alpha_{2}, \alpha_{3}, \alpha_{4}, \alpha_{5}, \alpha_{6}, \alpha_{7}, \alpha_{8}, \alpha_{9}\right]$. The algorithm will control the hand to grasp the object with the action function based on the configuration of the selected grasp topology. In this way, the robot is able adjust the grasp size while keep the basic configuration of the selected grasp topology.

We divide the hand into six parts: palm, index finger, middle finger, ring finger, little finger and thumb. The observation of this algorithm a vector with 12 dimensions. The first six dimensions of the vector is norm force provided by each part of the hand, and the last six dimensions indicates the closest distance between each part of the hand and the target object.

Since more contact points on the objects and minimize the total force are encouraged for a more steady grasp, we define the reward function as

$$
R= \begin{cases}-1000 & c=0 \\ \beta_{1} c-\beta_{2} f & c>0\end{cases}
$$

where $c$ is number of contact pints on target object, $f$ is total force, and $\beta$ is the scaler to control the weight of $c$ and $f$. In case a grasp is a success $R=R+1000$.

A simulation environment including a sawyer robotic arm, an AR10 hand and a workspace is also implemented to test the algorithm. A reset function is provide to reset the environment to its initial configuration at the beginning of an episode. A total of 15 pre-defined grasp topology was developed to grasp and move the target object. We define a grasp is a success when the hand is able to grasp the object and move the object above a certain height $(20 \mathrm{~cm}$ above original location). The episode is terminated when the robot have tried to grasp or run out of steps.

Training set contains 537 different grasp topology-object combinations. We also added a small noised (less than 5 $\mathrm{mm}$ ) to the object position to improve the adaptivity of the algorithm, and extend the training set to 5370. The network is trained with 0.00025 learning rate, 0.9 discount factor and $50 \mathrm{k}$ samples. We also added a small noised to the object position to improve the adaptivity of the algorithm. The accuracy on the training object achieved $88.2 \%$.

\section{EXPERIMENTS}

We conducted experiments to evaluate our grasp learning algorithm and to demonstrate its performance in a simulation environment. A video is also included to illustrate simulation results.

\section{A. Experiment Setup}

A simulation environment as shown in Fig. 5 is developed to evaluate the performance of the proposed algorithm. The environment includes a robot and a workplace. The robot contains an AR10 robotic hand and a sawyer robotic arm, which are attached together to deploy manipulation task. A table is placed in front of the robot as a work place. The objects are placed at a fix location in the workspace. The environment is developed with pybullet using python 3.5 in Ubuntu 16.04.

About 10\% (650) of the data from task-oriented object grasping dataset was selected from the dataset and used for 


\begin{tabular}{|c|c|c|c|c|c|c|c|c|c|c|c|}
\hline Object & A & B & C & Rigidity & Mass & Shape & Type & Task_group & Duration & Grasp & Grasp_group \\
\hline battery & 6 & 1 & 1 & rigid & 33.5 & prolate & cylinder & arranging & 0.64 & tripod & Precision pad abd 2-3 \\
\hline wrench & 15 & 2 & 0.75 & rigid & 250 & bladed & irregular & adjusting & 14.3 & thumb-index finger & Precision pad abd 2 \\
\hline rod & 15 & 1.25 & 1.25 & rigid & 300 & prolate & cylinder & adjusting & 3 & thumb-index finger & Precision pad abd 2 \\
\hline pliers & 10.5 & 5.5 & 0.75 & rigid & 100 & bladed & irregular & holding & 1 & lateral pinch & Intermediate add 2 \\
\hline calipers & 15 & 2.5 & 0.7 & rigid & 171 & bladed & irregular & moving & 4.5 & medium wrap & Power palm abd 2-5 \\
\hline calculator & 15 & 12 & 1 & rigid & 65 & oblate & short prisn & using & 1 & thumb-4 finger & Precision pad abd 2-5 \\
\hline sandpaper & 8 & 5.5 & 0.15 & squeezable & 10 & oblate & short prisn & ripping & 1 & lateral pinch & Intermediate add 2 \\
\hline torch & 15 & 9.5 & 9.5 & squeezable & 400 & prolate & cylinder & holding & 16.72 & large diameter & Power palm abd 2-5 \\
\hline saw & 10 & 4 & 2.5 & rigid & 600 & bladed & ellipse A & holding & 1 & light tool & Power palm add 2-5 \\
\hline marker & 14 & 1 & 1 & rigid & 15 & prolate & cylinder & holding & 2 & thumb-3 finger & Precision pad abd 2-4 \\
\hline goggles & 14 & 2 & 0.5 & rigid & 20 & bladed & irregular & putting & 3 & thumb-2 finger & Precision pad abd 2-3 \\
\hline mallet & 15 & 3 & 2.5 & rigid & 750 & prolate & cylinder & holding & 1 & index finger extension & Power palm add 3-5 \\
\hline metal can & 15 & 10 & 10 & rigid & 65 & prolate & cylinder & holding & 6 & large diameter & Power palm abd 2-5 \\
\hline tube & 15 & 1 & 1 & squeezable & 130 & prolate & cylinder & arranging & 1.96 & stick & Intermediate add 2 \\
\hline disk & 4.5 & 4.5 & 0.6 & rigid & 110 & oblate & disk & holding & 5 & lateral pinch & Intermediate add 2 \\
\hline handle & 12.5 & 2.25 & 2.25 & rigid & 300 & prolate & cylinder & pulling & 3 & thumb-3 finger & Precision pad abd 2-4 \\
\hline chuck key & 10 & 1 & 1 & rigid & 80 & prolate & long prism & picking up & 10 & medium wrap & Power palm abd 2-5 \\
\hline chuck & 10 & 1 & 1 & rigid & 50 & prolate & long prism & holding & 4 & medium wrap & Power palm abd 2-5 \\
\hline bit & 8.5 & 0.85 & 0.85 & rigid & 22.5 & prolate & cylinder & picking up & 9 & palmar & Power palm add 2-5 \\
\hline screwdriver & 15 & 3.5 & 3.5 & rigid & 100 & prolate & cylinder & moving & 2.8 & medium wrap & Power palm abd 2-5 \\
\hline rod-like part & 15 & 1 & 1 & rigid & 115 & prolate & cylinder & holding & 4.08 & lateral pinch & Intermediate add 2 \\
\hline hammer & 15 & 2 & 2 & rigid & 400 & prolate & cylinder & hammering & 1.64 & light tool & Power palm add 2-5 \\
\hline roll of tape & 6 & 6 & 1.5 & squeezable & 47.5 & oblate & disk & arranging & 0.44 & thumb-2 finger & Precision pad abd 2-3 \\
\hline thin rod & 11.5 & 0.65 & 0.65 & rigid & 105 & prolate & cylinder & turning & 8.76 & tripod & Precision pad abd 2-3 \\
\hline cabinet door & 15 & 15 & 1.75 & rigid & 1000 & oblate & short prisn & closing & 3.24 & palmar & Power palm add 2-5 \\
\hline lathe key & 9 & 1 & 1 & rigid & 65 & prolate & cylinder & adjusting & 11.6 & lateral pinch & Intermediate add 2 \\
\hline metal cylinder & 7.5 & 4.5 & 4.5 & rigid & 100 & prolate & cylinder & moving & 8.8 & lateral tripod & Intermediate add 3 \\
\hline safety glasses & 12.5 & 1 & 0.75 & fragile & 20 & prolate & cylinder & moving & 6.76 & tip pinch & Precision pad abd 2 \\
\hline oil bottle & 10 & 3.5 & 3.5 & squeezable & 125 & prolate & cylinder & oiling & 11.2 & thumb-4 finger & Precision pad abd 2-5 \\
\hline knob & 3 & 3 & 3 & rigid & 50 & equant & sphere & pushing & 0.12 & tripod & Precision pad abd 2-3 \\
\hline air hose & 15 & 3.25 & 1.75 & rigid & 231.5 & bladed & ellipse A & blowing & 11.9 & medium wrap & Power palm abd 2-5 \\
\hline plastic shield & 15 & 15 & 0.75 & rigid & 350 & oblate & short prisn & adjusting & 0.84 & palmar & Power palm add 2-5 \\
\hline thin parallel & 15 & 3.5 & 0.4 & rigid & 140 & bladed & irregular & holding & 5.76 & lateral pinch & Intermediate add 2 \\
\hline end mill & 11.5 & 0.75 & 0.75 & rigid & 65 & prolate & cylinder & holding & 1.2 & tripod & Precision pad abd 2-3 \\
\hline
\end{tabular}

Figure 4. Human grasping knowledge. Selected data samples in the task-object grasp dataset. Not all features are shown due to the size limitation of the Fig.

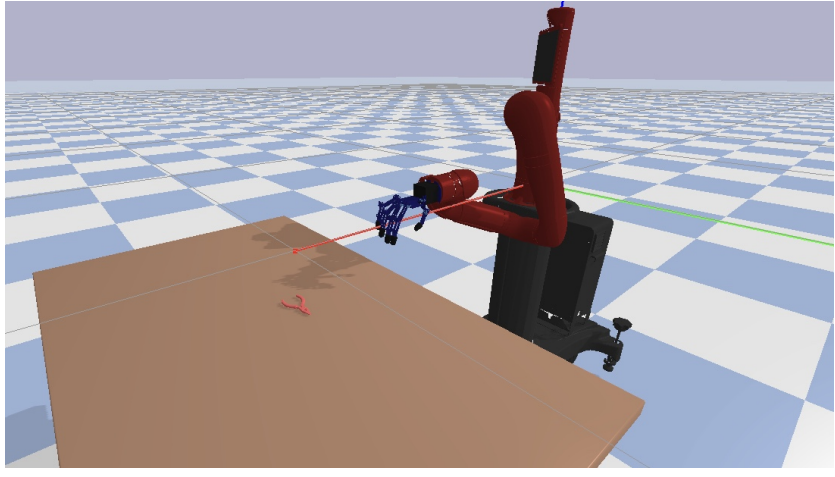

Figure 5. A simulation environment which allows complex grasping task.

testing. A random noise that follow the standard deviation was added to the continuous features of the remained data, and expand the size of training set to136627.

Not all $1573 \mathrm{~d}$ object models were used to train/test the reinforcement learning network. Some of the $3 \mathrm{D}$ objects are either to large for the workspace or too small to be grasped due to the physical configuration for robotic hand. $1033 \mathrm{D}$ objects were actually used in this work. 86 objects were used for training the adaptive grasp deployment network, and 17 objects were used for testing the algorithm.

\section{B. Grasp Strategies Learning}

The proposed grasp parameter learning network The network was tested with 650 new data samples. The accuracy on the OppoType selection network and the PIP selection network were $85.9 \%$ and $86.3 \%$, the confusion matrices are shown in Fig. 6. The result of grasp selection network achieved a hit rate of $100 \%$ and an overall top-3 match rate of $98.6 \%$. Recall, precision and F1 score of under different threshold is shown in Fig. 7. The testing accuracy were a little higher than the training accuracy. There are two reason that may lead to this result. Firstly, the training set is larger enough to cover most task-grasp combination. Secondly, there were only 650 testing samples in this experiment.

\section{Simulated Grasping of Arbitrary Objects}

Since the AR10 hand only has 10 DOF and can not perform some of the pre-dfined grasp topology, Only nine of the predefine grasp topology were actually tested in the algorithm. Each of the nine pre-define grasp topology was tested with three new objects, and each object was grasped 100 times. 


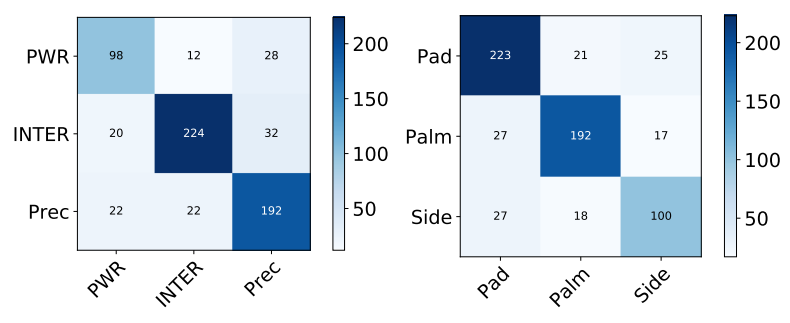

Figure 6. Confusion matrix of the PIP and OppoType selection network. PWR, INTER and Prec are short for Power, intermediate and Precision.

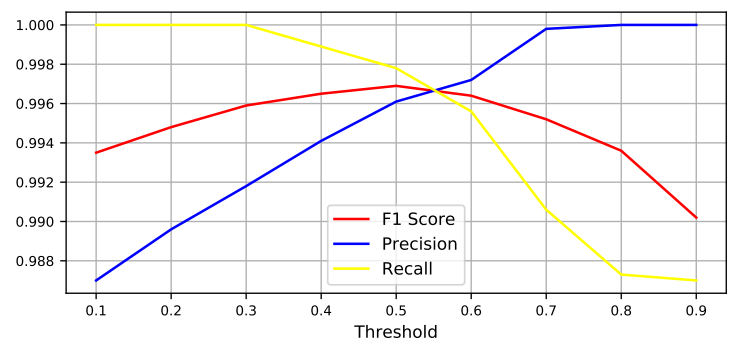

Figure 7. Precision, Recall and F1 score under different threshold.

A total of 17 objects and 2700 grasping were used to test the adaptive grasping learning system as shown in Fig. 8 and the corresponding results can be found in Table I. The results show 2312 successful grasping simulations with a success rate of $85.6 \%$ which was lower than training success rate.

Table I

RESULTS FOR SIMULATED GRASPING IN FIG. 8

\begin{tabular}{llll}
\hline Grasp Topology & \multicolumn{3}{l}{ Success Rate $\mathbf{( \% )}$} \\
\hline Power pad abd 2 & 94 & 93 & 85 \\
Power pad abd 2-4 & 91 & 82 & 75 \\
Power palm abd 2-5 & 89 & 83 & 78 \\
Power palm add 2-5 & 82 & 98 & 77 \\
Prec. pad abd 2 & 85 & 84 & 93 \\
Prec. pad abd 2-3 & 95 & 74 & 96 \\
Prec. pad abd 2-4 & 91 & 75 & 78 \\
Prec. pad abd 2-5 & 89 & 98 & 76 \\
Prec. pad add 2-5 & 82 & 78 & 91 \\
\hline
\end{tabular}

There were some reasons that may lead this result. The first one is that the object is completely new for the algorithm which brought difficulties for the robot to grasping. The robot hand is not dexterous enough for certain objects. For example, while using Prec. pad abd 2-5 to grasp a wrench Fig. 8, the success rate is low due to the thin shape of the wrench. Finally, the $3 \mathrm{~d}$ object models are not perfect and some of the models may not accuracy enough. For example, for the banana in Fig. 8, we can clearly see that the shape collision model of the banana is not the same as the visual model.

\section{CONCLUSIONS}

We presented a grasp learning algorithm which is able to learn grasp topology from human knowledge and deploy the grasp adaptively. A multi-output, multi-label neutral network was developed to predict grasp topology based on tasks. The top 1 accuracy for this network achieved 100\%. A Proximal Policy Optimization reinforcement learning algorithm is trained for adaptive grasp learning. A total of 2700 grasping experiments were performed on different objects and the accuracy achieved $85.6 \%$. The performance of the system was demonstrated by the experiment results.

\section{REFERENCES}

[1] K. Zhou, G. Ebenhofer, C. Eitzinger, U. Zimmermann, C. Walter, J. Saenz, L. P. Castaño, M. A. F. Hernández, and J. N. Oriol, "Mobile manipulator is coming to aerospace manufacturing industry," in 2014 IEEE International Symposium on Robotic and Sensors Environments (ROSE) Proceedings. IEEE, 2014, pp. 94-99.

[2] P. Božek, "Robot path optimization for spot welding applications in automotive industry," Tehnicki Vjesnik-Technical Gazette, vol. 20, no. 5, pp. 913-917, 2013.

[3] S. Mitsi, K.-D. Bouzakis, G. Mansour, D. Sagris, and G. Maliaris, "Off-line programming of an industrial robot for manufacturing," The International Journal of Advanced Manufacturing Technology, vol. 26, no. 3, pp. 262-267, 2005.

[4] S. Torres-Mendez and A. Khajepour, "Design optimization of a warehousing cable-based robot," in International Design Engineering Technical Conferences and Computers and Information in Engineering Conference, vol. 46360. American Society of Mechanical Engineers, 2014, p. V05AT08A091.

[5] T. Feix, J. Romero, H.-B. Schmiedmayer, A. M. Dollar, and D. Kragic, "The grasp taxonomy of human grasp types," IEEE Transactions on Human-Machine Systems, vol. 46, no. 1, pp. 66-77, 2016.

[6] M. R. Cutkosky et al., "On grasp choice, grasp models, and the design of hands for manufacturing tasks." IEEE Transactions on robotics and automation, vol. 5, no. 3, pp. 269-279, 1989.

[7] I. M. Bullock, T. Feix, and A. M. Dollar, "The yale human grasping dataset: Grasp, object, and task data in household and machine shop environments," The International Journal of Robotics Research, vol. 34, no. 3, pp. 251-255, 2015.

[8] T. Feix, I. M. Bullock, and A. M. Dollar, "Analysis of human grasping behavior: Object characteristics and grasp type," IEEE transactions on haptics, vol. 7, no. 3, pp. 311-323, 2014.

[9] B. Rao, "Learning robotic grasping strategy based on natural-language object descriptions," 2018.

[10] H. Li, J. Tan, and H. He, "Magichand: Context-aware dexterous grasping using an anthropomorphic robotic hand," in 2020 IEEE International Conference on Robotics and Automation (ICRA). IEEE, 2020, pp. 9895-9901.

[11] J. Schulman, F. Wolski, P. Dhariwal, A. Radford, and O. Klimov, "Proximal policy optimization algorithms," arXiv preprint arXiv:1707.06347, 2017.

[12] V. R. Konda and J. N. Tsitsiklis, "Actor-critic algorithms," in Advances in neural information processing systems, 2000, pp. 1008-1014. 
CONFIDENTIAL. Limited circulation. For review only.

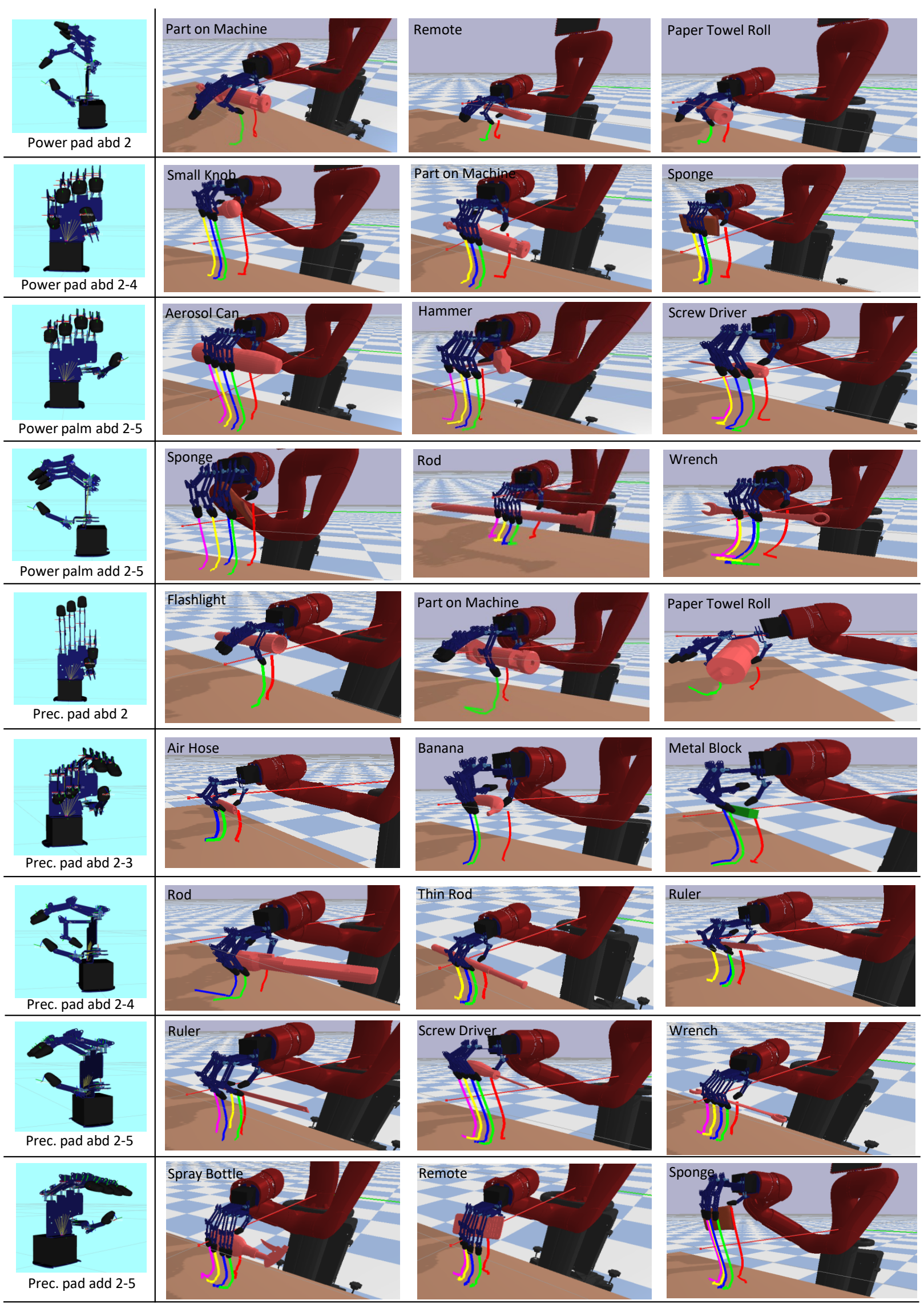

Figure 8. Grasping simulations on 17 arbitrary objects, colored lines indicate trajectory of finger tips while grasping. 